TRANSACTIONS OF THE

AMERICAN MATHEMATICAL SOCIETY

Volume 358, Number 7 , Pages 2897-2916

S 0002-9947(05)04012-2

Article electronically published on December 20, 2005

\title{
QUILLEN STRATIFICATION FOR HOCHSCHILD COHOMOLOGY OF BLOCKS
}

\author{
JONATHAN PAKIANATHAN AND SARAH WITHERSPOON, \\ AND WITH AN APPENDIX BY STEPHEN F. SIEGEL
}

\begin{abstract}
We decompose the maximal ideal spectrum of the Hochschild cohomology ring of a block of a finite group into a disjoint union of subvarieties corresponding to elementary abelian $p$-subgroups of a defect group. These subvarieties are described in terms of group cohomological varieties and the Alperin-Broué correspondence on blocks. Our description leads in particular to a homeomorphism between the Hochschild variety of the principal block and the group cohomological variety. The proofs require a result of Stephen F. Siegel, given in the Appendix, which states that nilpotency in Hochschild cohomology is detected on elementary abelian $p$-subgroups.
\end{abstract}

\section{INTRODUCTION}

Quillen's two groundbreaking papers of 1971 [18] yield a description of the variety of a finite group $G$, that is, of the maximal ideal spectrum of the cohomology ring $\mathrm{H}^{*}(G, k)$, as a disjoint union of subvarieties corresponding to elementary abelian $p$-subgroups (where $p$ is the characteristic of the commutative ring $k$ ). Avrunin and Scott vastly generalized this result to varieties of modules over the group ring $k G$, that is, to the maximal ideal spectra of quotients of $\mathrm{H}^{*}(G, k)$ associated to these modules [5]. In this paper we replace $\mathrm{H}^{*}(G, k)$ by the Hochschild cohomology ring $\mathrm{HH}^{*}(k G)$ of the group algebra $k G$, and find a suitable analog of the Quillen stratification, describing the maximal ideal spectrum of $\operatorname{HH}^{*}(k G)$ as a disjoint union of varieties corresponding to elementary abelian $p$-subgroups. As this Hochschild cohomology decomposes according to the decomposition of $k G$ into blocks (that is, indecomposable ideal direct summands), this description is given by a stratification of the Hochschild cohomology ring $\operatorname{HH}^{*}(B)$ of each block $B$. This theory is parallel to that of Linckelmann [16], in which he gives a Quillen stratification for the maximal ideal spectrum of the block cohomology ring $\operatorname{LH}^{*}(B)$ of a block $B$, where $\mathrm{LH}^{*}(B)$ is a particular subring of the group cohomology ring of a defect group of $B$. However the techniques we develop to work with Hochschild cohomology are quite different from those of Linckelmann, and begin with a particular ring homomorphism (Theorem 2.5 below).

The relationship between the Hochschild cohomology ring $\operatorname{HH}^{*}(B)$ and Linckelmann's block cohomology ring $\operatorname{LH}^{*}(B)$ is not fully understood. In the case of the principal block, our results imply that their respective varieties are homeomorphic.

Received by the editors March 3, 2004.

2000 Mathematics Subject Classification. Primary 20J06.

The second author was supported by National Security Agency Grant \#MDS904-01-1-0067 and National Science Foundation Grant \#DMS0245560. 
The general case is more complicated, but is potentially where the main significance of these cohomology theories lies: Blocks other than the principal block are not augmented algebras themselves, and so one must use a cohomology theory such as Hochschild cohomology or Linckelmann's block cohomology to study them. There are a number of recent papers that use Hochschild cohomology to study various types of finite-dimensional algebras and their modules, for example see [10, 13, 24]. Theories of support varieties for modules have been built from Hochschild cohomology [21, 24] and from Linckelmann's block cohomology [16]. It is hoped that the present paper will lead to a better understanding of the connections between these two cohomology theories for blocks of finite groups and their modules. This would augment our knowledge of both and give some insight into any connection between the Morita structure (related to Hochschild cohomology) and the local structure (related to block cohomology) of a block.

We will start by summarizing the main results of this paper in greater detail. From now on $k$ will be an algebraically closed field of positive characteristic $p$ dividing the order of the finite group $G$. The Hochschild cohomology ring $\operatorname{HH}^{*}(k G)=$ $\operatorname{Ext}_{k G \otimes(k G)^{o p}}^{*}(k G, k G)$ is known to be isomorphic to $\mathrm{H}^{*}(G, k G)=\operatorname{Ext}_{k G}^{*}(k, k G)$, where $G$ acts on $k G$ via conjugation. This observation goes back to Eilenberg and Mac Lane, however the proof that the ring structures correspond appears in 22, $\S 3]$. The ring structure on $\mathrm{H}^{*}(G, k G)$ is given by the cup product followed by the algebra multiplication map $k G \otimes_{k} k G \rightarrow k G$. Similarly, if $B$ is a block of $k G$, then $\mathrm{HH}^{*}(B) \cong \mathrm{H}^{*}(G, B)$, where the action of $G$ on $B$ is via conjugation. In this paper, we will work almost exclusively with the rings $\mathrm{H}^{*}(G, k G)$ and $\mathrm{H}^{*}(G, B)$ in order to exploit group cohomological techniques.

It is known that $\mathrm{HH}^{*}(k G)$ is a finitely generated, graded-commutative algebra. Thus the maximal ideal spectrum of $\operatorname{HH}^{*}(k G)$ (equivalently, of its even degree subring when $p \neq 2$ ) is an affine algebraic variety which we denote by $X_{G}$. The details, and the following theorems, appear in Section 2. If $H$ is a subgroup of $G$, let $C_{G}(H)$ denote the centralizer of $H$ in $G$, that is, $C_{G}(H)=\{g \in G \mid g h=$ $h g$ for all $h \in H\}$.

Theorems 2.5, 2.10. There is a ring homomorphism, with nilpotent kernel,

$$
\mathrm{HH}^{*}(k G) \rightarrow \prod_{E}\left(k C_{G}(E) \otimes_{k} \mathrm{H}^{*}(E, k)\right)^{N_{G}(E)},
$$

the product over a set of representatives $E$ of conjugacy classes of elementary abelian p-subgroups $E$ of $G$. Thus the Hochschild variety $X_{G}$ of $k G$ is a union of subvarieties $X_{G, E}$, the union taken over the same set.

The structure of the subvarieties $X_{G, E}$ in the theorem is given roughly by the existence of a finite surjection $Y_{G, E} \rightarrow X_{G, E}$, where $Y_{G, E}$ is a disjoint union of affine varieties of dimension equal to the rank of $E$, the union taken over the set of blocks of $k C_{G}(E)$.

In order to refine this description, we consider the Hochschild variety of a block $B$ of $k G$. Let $k G \cong B_{1} \times \cdots \times B_{n}$ be the decomposition into blocks (that is, indecomposable ideal factors) $B_{1}, \ldots, B_{n}$. As there are no nonzero $k G$-bimodule maps between distinct blocks, $\mathrm{HH}^{*}(k G) \cong \mathrm{HH}^{*}\left(B_{1}\right) \times \cdots \times \mathrm{HH}^{*}\left(B_{n}\right)$ as algebras. The Hochschild variety $X_{G}$ of $k G$ is thus the disjoint union of the Hochschild varieties $X_{B}$ of its blocks $B$, where $X_{B}$ is the maximal ideal spectrum of $\mathrm{HH}^{*}(B) \cong$ $\mathrm{H}^{*}(G, B)$. We again find that each $X_{B}$ is a union of subvarieties $X_{B, E}$, where this 
time $E$ ranges over a set of $G$-conjugacy representatives of elementary abelian $p$ subgroups of a defect group of $B$. There are finite surjective maps $Y_{B, E} \rightarrow X_{B, E}$, where $Y_{B, E}$ is a disjoint union of affine varieties of dimension equal to the rank of $E$, the union taken over the set of blocks of $k C_{G}(E)$ corresponding to $B$ under the Alperin-Broué correspondence. In the union $X_{B}=\bigcup_{E} X_{B, E}$, we must determine how the pieces are glued together to form $X_{B}$. In contrast to group cohomology, $X_{B, F}$ does not necessarily inject into $X_{B, E}$ when $F<E$. Thus we find it necessary to introduce auxiliary varieties $X_{B, E, F}$ with maps $X_{B, F} \stackrel{\mathrm{Br}^{*}}{\longleftarrow} X_{B, E, F} \hookrightarrow X_{B, E}$ that provide the gluing information for $X_{B}$. The map $\mathrm{Br}^{*}$ is given by the Alperin-Broué correspondence and is neither injective nor surjective in general.

Let $X_{B, E}^{+}=X_{B, E}-\bigcup_{F<E} X_{B, E, F}$, the union over all proper subgroups $F$ of $E$, and define $Y_{B, E}^{+}$similarly. As before, there are finite surjective maps $Y_{B, E}^{+} \rightarrow X_{B, E}^{+}$. Let $W_{G}(E)=N_{G}(E) / C_{G}(E)$, the Weyl group of $E$. The following result depends on some technical work done in Section 3:

Theorems 4.2, 4.3 (Quillen stratification). Let $B$ be a block of $G$ and $P$ a defect group of $B$. The Hochschild variety $X_{B}$ of $B$ is a disjoint union of subvarieties $X_{B, E}^{+}$, the union over a set of elementary abelian p-subgroups $E$ of $P$, one from each $G$-conjugacy class of elementary abelian p-subgroups of $G$ for which at least one member is contained in $P$. If $E$ is an elementary abelian p-subgroup of $P$ for which there is a unique block of $k C_{G}(E)$ corresponding to $B$ under the AlperinBroué correspondence, then there is an inseparable isogeny $Y_{B, E}^{+} / W_{G}(E) \rightarrow X_{B, E}^{+}$.

The varieties $Y_{B, E}^{+}$of the theorem are given explicitly in terms of group cohomological varieties and the Alperin-Broué correspondence. The last statement applies in particular to the principal block $B_{0}$, and in this special case we have the following corollary.

Corollary 4.5. The Hochschild variety $X_{B_{0}}$ of the principal block $B_{0}$ is homeomorphic to the maximal ideal spectrum of the group cohomology ring $\mathrm{H}^{*}(G, k)$.

This proves a weak form of the conjecture that $\mathrm{HH}^{*}\left(B_{0}\right)$ is isomorphic to $\mathrm{H}^{*}(G, k)$, modulo their radicals [23, Conj. 1] (see also [22, §11, Question 2]). A more general question, motivated by the results of [17], involves Linckelmann's block cohomology $\mathrm{LH}^{*}(B)$ (see Definition 4.6):

Question 4.7. Let $B$ be a block of $k G$. Are the maximal ideal spectra of $\operatorname{HH}^{*}(B)$ and $\mathrm{LH}^{*}(B)$ homeomorphic?

In the case of the principal block $B_{0}$, it is known that $\operatorname{LH}^{*}\left(B_{0}\right) \cong \mathrm{H}^{*}(G, k)$, and so Corollary 4.5 gives an affirmative answer to this question. Our paper [17] also gives a positive answer in the cases (1) $B$ has a cyclic defect group and (2) $G$ is a Frobenius group ( $p$ odd). In fact we proved the stronger result that $\mathrm{HH}^{*}(B)$ and $\mathrm{LH}^{*}(B)$ are isomorphic, modulo their radicals, in these cases. We hope that the results of the present paper will ultimately lead to an answer to this question in general. Such an answer could have important consequences in block theory.

The Appendix, written by Stephen F. Siegel, contains a detection result that is needed for our Quillen stratification. Hochschild cohomology is not a functor on algebras. However, if the algebra is a group algebra, we may define restriction maps to subgroup algebras by keeping coefficients in the original group ring. Then nilpotent elements are detected by restrictions to elementary abelian $p$-subgroups, 
as stated in Proposition 2.1 in the next section. The proof is similar to the standard proof given for the analogous result in group cohomology, but there are some subtleties involved. This detection result is a special case of the following more general result in the Appendix.

Lemma 5.1. Let $A$ be an algebra on which $G$ acts by automorphisms, and $M$ an A-module that is also a $k G$-module for which these two module structures are compatible. Let $\zeta \in \mathrm{H}^{*}(G, A)$ such that for each elementary abelian p-subgroup $E$ of $G$, some power of $\operatorname{res}_{E}^{G}(\zeta) \in \mathrm{H}^{*}(E, A)$ annihilates $\mathrm{H}^{*}(E, M)$. Then some power of $\zeta$ annihilates $\mathrm{H}^{*}(G, M)$.

The detection result we need is a consequence of Lemma 5.1 and is also a special case of a theorem of Suslin for finite group schemes 25]. To be complete and to allow for potential applications of Lemma 5.1 itself, we have decided to include the details of the proof in the Appendix.

\section{The Hochschild VARIETy of $G$}

We will begin with some results about the ring structure of $\operatorname{HH}^{*}(k G) \cong \mathrm{H}^{*}(G, k G)$ (where the action of $G$ on $k G$ is via conjugation) and their consequences for the structure of its maximal ideal spectrum. First we note that $\mathrm{H}^{*}(G, k G)$ is gradedcommutative by a very general result of Gerstenhaber [12, $\S 7$, Cor. 1]. It is also finitely generated: There is an embedding of the group cohomology ring $\mathrm{H}^{*}(G, k)$ into $\mathrm{H}^{*}(G, k G)$ induced by the unit map $k \hookrightarrow k G$. Now $\mathrm{H}^{*}(G, k G)$ is finitely generated as an $\mathrm{H}^{*}(G, k)$-module, and $\mathrm{H}^{*}(G, k)$ is finitely generated [11, Thm. 7.4.1, Cor. 7.4.6]. The union of two such sets of generators yields a set of generators for $\mathrm{H}^{*}(G, k G)$.

The following result on nilpotent elements in $\mathrm{H}^{*}(G, k G)$ is also stated as Corollary 5.4, proved in the Appendix. Alternatively, the proposition follows from a very general theorem of Suslin for finite group schemes 25. If $H<G$, we will use the notation $\operatorname{res}_{H}^{G}$ and $\operatorname{cor}_{H}^{G}$ for the restriction and corestriction maps, respectively. (See [6] or [1] for details.)

Proposition 2.1. Let $\zeta \in \mathrm{H}^{*}(G, k G)$. Then $\zeta$ is nilpotent if, and only if, res ${ }_{E}^{G}(\zeta)$ is nilpotent for every elementary abelian p-subgroup $E$ of $G$.

For any subgroup $H$ of $G$, let $\overline{\mathrm{H}}^{*}(H, k G)$ (respectively, $\left.\overline{\mathrm{H}}^{*}(H, k)\right)$ denote the quotient of the cohomology ring $\mathrm{H}^{*}(H, k G)$ (respectively, $\left.\mathrm{H}^{*}(H, k)\right)$ by the ideal of "proper transfers" $\sum_{K<H} \operatorname{Im}\left(\operatorname{cor}_{K}^{H}\right)$. We will be interested in the ring homomorphism

$$
\sigma: \mathrm{H}^{*}(G, k G) \rightarrow \prod_{E}\left(\overline{\mathrm{H}}^{*}(E, k G)\right)^{N_{G}(E)},
$$

the product over a set of representatives $E$ of conjugacy classes of elementary abelian $p$-subgroups of $G$, given in the $E$-component by the composition $\overline{r e s}_{E}^{G}$ of $\operatorname{res}_{E}^{G}$ with the quotient map. We will use the following result from 22]. Statement (i) in the proposition below is essentially the Universal Coefficients Theorem [8, VI, Thm. 3.3], whereas the proof of (ii) is more involved and uses a product formula for Hochschild cohomology [22, Thm. 5.1].

Proposition 2.3. Let $H$ be a subgroup of $G$, acting on $k G$ by conjugation. The following are isomorphisms of graded algebras. 
(i) 22, Prop. 3.2] $\mathrm{H}^{*}\left(H, k C_{G}(H)\right) \cong k C_{G}(H) \otimes_{k} \mathrm{H}^{*}(H, k)$.

(ii) [22, Thm. 10.2] $\overline{\mathrm{H}}^{*}(H, k G) \cong k C_{G}(H) \otimes_{k} \overline{\mathrm{H}}^{*}(H, k)$.

For an elementary abelian $p$-group $E$, we have $\overline{\mathrm{H}}^{*}(E, k)=\mathrm{H}^{*}(E, k)$ [11, Lem. 6.3.4], so part (ii) of the above proposition implies that there is a ring isomorphism

$$
\psi_{E}: \overline{\mathrm{H}}^{*}(E, k G) \stackrel{\sim}{\longrightarrow} k C_{G}(E) \otimes_{k} \mathrm{H}^{*}(E, k) .
$$

Part (i) further implies that $k C_{G}(E) \otimes_{k} \mathrm{H}^{*}(E, k) \cong \mathrm{H}^{*}\left(E, k C_{G}(E)\right)$. We will sometimes consider $\psi_{E}$ to be a map to $\mathrm{H}^{*}\left(E, k C_{G}(E)\right)$ when it is convenient. Let $\rho$ be the composition of $\sigma$ with the direct product of these isomorphisms $\psi_{E}$. Another way to view $\rho$ is as the product of restriction maps $\operatorname{res}_{E}^{G}$, each followed by the map induced by the projection of $k G=k C_{G}(E) \times k\left(G-C_{G}(E)\right)$ onto the first factor, and then by the isomorphism of part (i) of the above proposition.

Theorem 2.5. There is a ring homomorphism, with nilpotent kernel,

$$
\rho: \mathrm{H}^{*}(G, k G) \rightarrow \prod_{E}\left(k C_{G}(E) \otimes_{k} \mathrm{H}^{*}(E, k)\right)^{N_{G}(E)},
$$

the product over a set of representatives $E$ of conjugacy classes of elementary abelian p-subgroups of $G$.

Proof. As $\rho$ is the composition of $\sigma$ (2.2) with the isomorphisms $\psi_{E}$ (2.4), it suffices to prove that $\operatorname{Ker}(\sigma)$ is nilpotent. This is equivalent to showing that $\operatorname{Ker}(\sigma)$ consists entirely of nilpotent elements, as $\mathrm{H}^{*}(G, k G)$ is graded-commutative.

Let $\zeta \in \operatorname{Ker}(\sigma)$. Then for each $E$ in the product, $\operatorname{res}_{E}^{G}(\zeta) \in \sum_{F<E} \operatorname{Im}\left(\operatorname{cor}_{F}^{E}\right)$. We will show that $\operatorname{res}_{E}^{G}(\zeta)$ is nilpotent; this involves an argument similar to the proof of [26, Thm. 3.2, part 2], but we repeat it here for completeness. It will follow that $\zeta$ is nilpotent by Proposition 2.1. as each elementary abelian $p$-subgroup of $G$ is conjugate to one of the subgroups $E$ in the product, and $\operatorname{res}_{E}^{G}(\zeta)$ is nilpotent if and only if its conjugates by all elements of $G$ are nilpotent.

Write $\operatorname{res}_{E}^{G}(\zeta)=\sum_{F<E} \operatorname{cor}_{F}^{E}\left(\zeta_{F}\right)$ for some elements $\zeta_{F} \in \mathrm{H}^{*}(F, k G)$. Then

$$
\left(\operatorname{res}_{E}^{G}(\zeta)\right)^{2}=\operatorname{res}_{E}^{G}(\zeta) \cdot \sum_{F<E} \operatorname{cor}_{F}^{E}\left(\zeta_{F}\right)=\sum_{F<E} \operatorname{cor}_{F}^{E}\left(\operatorname{res}_{F}^{G}(\zeta) \cdot \zeta_{F}\right) .
$$

As $\zeta \in \operatorname{Ker}(\sigma)$, we also have $\operatorname{res}_{F}^{G}(\zeta)=\sum_{L<F} \operatorname{cor}_{L}^{F}\left(\zeta_{L}\right)$ for some elements $\zeta_{L} \in$ $\mathrm{H}^{*}(L, k G)$. Therefore

$$
\begin{aligned}
\left(\operatorname{res}_{E}^{G}(\zeta)\right)^{2} & =\sum_{F<E} \operatorname{cor}_{F}^{E}\left(\sum_{L<F} \operatorname{cor}_{L}^{F}\left(\zeta_{L}\right) \cdot \zeta_{F}\right) \\
& =\sum_{F<E} \sum_{L<F} \operatorname{cor}_{L}^{E}\left(\zeta_{L} \cdot \operatorname{res}_{L}^{F}\left(\zeta_{F}\right)\right) .
\end{aligned}
$$

Similarly, we may calculate $\left(\operatorname{res}_{F}^{G}(\zeta)\right)^{3}$, and so on. The sizes of the subgroups involved in the power $\left(\operatorname{res}_{E}^{G}(\zeta)\right)^{n}$ strictly decreases as $n$ increases, and so $\left(\operatorname{res}_{E}^{G}(\zeta)\right)^{n}=$ 0 for some $n$.

We may use the map $\rho$ in the theorem to obtain a result regarding the maximal ideal spectrum of the Hochschild cohomology ring $\mathrm{H}^{*}(G, k G)$, where

$$
\mathrm{H}^{\bullet}(G, k G)=\left\{\begin{array}{l}
\mathrm{H}^{2 *}(G, k G), \text { if } p \text { is odd } \\
\mathrm{H}^{*}(G, k G), \text { if } p=2 .
\end{array}\right.
$$


The ring $\mathrm{H}^{*}(G, k G)$ is commutative, as $\mathrm{H}^{*}(G, k G)$ is graded-commutative. The map $\rho$ restricted to $\mathrm{H}^{*}(G, k G)$ will also be denoted $\rho$.

For each elementary abelian $p$-subgroup $E$, the corresponding factor in the target of $\rho$ consists of elements invariant under $N_{G}(E)$, and so is contained in the invariants under $C_{G}(E)$ :

$$
\left(k C_{G}(E) \otimes_{k} \mathrm{H}^{\bullet}(E, k)\right)^{N_{G}(E)} \subseteq\left(k C_{G}(E) \otimes_{k} \mathrm{H}^{\bullet}(E, k)\right)^{C_{G}(E)} .
$$

However $C_{G}(E)$ acts trivially on $\mathrm{H}^{\bullet}(E, k)$, so the latter ring is $Z\left(k C_{G}(E)\right) \otimes_{k}$ $\mathrm{H}^{*}(E, k)$, a commutative algebra. We will set

$$
A^{\bullet}(E)=Z\left(k C_{G}(E)\right) \otimes_{k} \mathrm{H}^{\bullet}(E, k),
$$

and define the algebra map

$$
\phi_{E}: \mathrm{H}^{\bullet}(G, k G) \rightarrow A^{\bullet}(E)
$$

by restriction of the map $\psi_{E} \circ \overline{r e s}_{E}^{G}$ to $\mathrm{H}^{\bullet}(G, k G)$, followed by inclusion into $A^{\bullet}(E)$ ( $\psi_{E}$ is defined in (2.4) and $\overline{r e s}_{E}^{G}$ in the text above (2.4) ).

We now define several varieties.

Definition 2.8. The Hochschild variety of $G$ is $X_{G}=\max \left(\mathrm{H}^{*}(G, k G)\right)$, the maximal ideal spectrum of $\mathrm{H}^{*}(G, k G)$. Let $Y_{G, E}=\max \left(A^{*}(E)\right)$ and $X_{G, E}=\max \left(\operatorname{Im}\left(\phi_{E}\right)\right)$. Note that $\phi_{E}$ induces a map $\phi_{E}^{*}: Y_{G, E} \rightarrow X_{G, E} \hookrightarrow X_{G}$.

We first describe an approximate relationship between the varieties $Y_{G, E}$ and $X_{G, E}$.

Lemma 2.9. The algebra $A^{\bullet}(E)$ is finitely generated over $\operatorname{Im}\left(\phi_{E}\right)$, so that $\phi_{E}^{*}$ : $Y_{G, E} \rightarrow X_{G, E}$ is a finite surjective map. In particular, $\operatorname{dim}\left(X_{G, E}\right)=\operatorname{dim}\left(Y_{G, E}\right)$.

Proof. It suffices to show that $k C_{G}(E) \otimes_{k} \mathrm{H}^{\bullet}(E, k)$ is finitely generated over the image of $\phi_{E}$, as $A^{\bullet}(E)=Z\left(k C_{G}(E)\right) \otimes_{k} \mathrm{H}^{\bullet}(E, k)$ is a submodule of this, and $\mathrm{H}^{*}(G, k G)$ is Noetherian.

Applying the isomorphism (2.4), we need to show that $\overline{\mathrm{H}}^{*}(E, k G)$ is finitely generated as a module over $\operatorname{res}_{E}^{G}\left(\mathrm{H}^{\bullet}(G, k G)\right)$, which will follow once we show that $\mathrm{H}^{*}(E, k G)$ is finitely generated over $\operatorname{res}_{E}^{G}\left(\mathrm{H}^{\bullet}(G, k G)\right)$. Now $\mathrm{H}^{\bullet}(E, k G)$ is finitely generated over $\mathrm{H}^{*}(E, k)$ by [11, Thm. 7.4.1], and $\mathrm{H}^{*}(E, k)$ is finitely generated over $\operatorname{res}_{E}^{G}\left(\mathrm{H}^{\bullet}(G, k)\right)$ by [11, Cor. 7.4.7]. Therefore $\mathrm{H}^{\bullet}(E, k G)$ is finitely generated over $\operatorname{res}_{E}^{G}\left(\mathrm{H}^{\bullet}(G, k)\right)$. (Take as a set of generators the set of pairwise products of the previous generators.) But $\operatorname{res}_{E}^{G}\left(\mathrm{H}^{*}(G, k)\right) \subseteq \operatorname{res}_{E}^{G}\left(\mathrm{H}^{*}(G, k G)\right)$, as these restriction maps are defined compatibly, and so $\mathrm{H}^{*}(E, k G)$ is finitely generated over $\operatorname{res}_{E}^{G}\left(\mathrm{H}^{*}(G, k G)\right)$. Thus $\phi_{E}^{*}$ is a finite map [7. p. 171]. As $\operatorname{Im}\left(\phi_{E}\right)$ is a subalgebra of $A^{\bullet}(E), \phi_{E}^{*}: Y_{G, E} \rightarrow X_{G, E}$ is surjective by the Going-up Theorem [4, Thm. 5.10]. It also follows from finiteness that $\operatorname{dim}\left(X_{G, E}\right)=\operatorname{dim}\left(Y_{G, E}\right)$.

As a consequence of the lemma, we may describe the relationship of the Hochschild variety $X_{G}$ to the varieties $X_{G, E}$ and $Y_{G, E}$ as follows.

Theorem 2.10. The Hochschild variety is a union,

$$
X_{G}=\bigcup_{E} \phi_{E}^{*}\left(Y_{G, E}\right)=\bigcup_{E} X_{G, E}
$$

taken over a set of representatives $E$ of conjugacy classes of elementary abelian p-subgroups of $G$. 
Proof. By Theorem 2.5, the map

$$
\prod_{E} \phi_{E}: \mathrm{H}^{\bullet}(G, k G) \rightarrow \prod_{E} A^{\bullet}(E)
$$

has nilpotent kernel. Since the product is finite, Lemma2.9 implies that the target of the map $\prod_{E} \phi_{E}$ is finitely generated as a module over its image. It follows from these two facts that

$$
\bigcup_{E} \phi_{E}^{*}: \bigcup_{E} Y_{G, E} \rightarrow X_{G}
$$

is a finite dominant map [7, p. 172]. Again by the Going-up Theorem 4, Thm. 5.10], $\bigcup_{E} \phi_{E}^{*}$ is surjective.

\section{The varieties $X_{G, E}$ And $Y_{G, E}$}

In this section, we work to better understand the varieties $X_{G, E}$ and $Y_{G, E}$ appearing in Theorem 2.10. First, $Y_{G, E}$ clearly depends on the block structure of the group algebra $k C_{G}(E)$, and accordingly we choose some notation.

Definition 3.1. If $E$ is an elementary abelian p-subgroup of $G$, denote by $\mathfrak{B}_{E}$ the set of blocks (that is, block ideals) of $k C_{G}(E)$. Thus $k C_{G}(E)=\prod_{b \in \mathfrak{B}_{E}} b$ as an algebra. Denote the block idempotent associated to the block $b$ by $e_{b}$, so that $b=k C_{G}(E) e_{b}$. For each block $b \in \mathfrak{B}_{E}$, let $Y_{b}=k^{\mathrm{rk}(E)}$, the affine space of dimension equal to the rank of $E$.

The following result gives a simple picture of the varieties $Y_{G, E}$.

Lemma 3.2. If $E$ is an elementary abelian p-subgroup of $G$, then

$$
Y_{G, E} \cong \bigcup_{b \in \mathfrak{B}_{E}} Y_{b}
$$

the disjoint union of affine spaces of dimension $\operatorname{rk}(E)$, one for each block of $k C_{G}(E)$.

Proof. By definition, $Y_{G, E}=\max \left(Z\left(k C_{G}(E)\right) \otimes_{k} \mathrm{H}^{*}(E, k)\right)$. If we write $k C_{G}(E)=$ $\prod_{b \in \mathfrak{B}_{E}} b$, then $Z\left(k C_{G}(E)\right)=\prod_{b \in \mathfrak{B}_{E}} Z(b)$, where $Z(b)$ is the center of the block $b$. Note that $Z(b) / \operatorname{rad}(Z(b)) \cong k$, generated by the image of $e_{b}$ [9, Prop. 56.16].

Now we may write $A^{\bullet}(E)=\prod_{b \in \mathfrak{B}_{E}}\left(Z(b) \otimes_{k} \mathrm{H}^{\bullet}(E, k)\right)$. Thus $Y_{G, E}$ is the disjoint union of the maximal ideal spectra of the summands $Z(b) \otimes_{k} \mathrm{H}^{*}(E, k)$. Since $Z(b) \otimes_{k}$ $\mathrm{H}^{\bullet}(E, k)$ is isomorphic, modulo radicals, to $Z(b) / \operatorname{rad}(Z(b)) \otimes_{k} \mathrm{H}^{\bullet}(E, k)$, its maximal ideal spectrum is

$$
\max \left(Z(b) / \operatorname{rad}(Z(b)) \otimes_{k} \mathrm{H}^{\bullet}(E, k)\right)=\max \left(\mathrm{H}^{\bullet}(E, k)\right) \cong k^{\mathrm{rk}(E)}=Y_{b} .
$$

The picture of the varieties $Y_{G, E}$ provided by the lemma gives us some understanding of the subvarieties $X_{G, E}$ of $X_{G}$, as each $\phi_{E}^{*}: Y_{G, E} \rightarrow X_{G, E}$ is a finite surjective map. We will work to refine this picture, and to understand how the varieties $X_{G, E}$ are glued together in the union of Theorem 2.10. For this, we will need the Brauer map. Let $F<E$ be elementary abelian $p$-subgroups of $G$, and note that

$$
F<E \leq C_{G}(E) \leq C_{G}(F) .
$$

The corresponding Brauer map is the linear function

$$
\operatorname{br}_{F}^{E}: Z\left(k C_{G}(F)\right) \rightarrow Z\left(k C_{G}(E)\right)
$$


defined by $\operatorname{br}_{F}^{E}(x)=x$ if $x \in C_{G}(E)$, and $\operatorname{br}_{F}^{E}(x)=0$ if $x \in C_{G}(F)-C_{G}(E)$. This is in fact an algebra homomorphism by [1, (2.5)(3)] or [9, Lemma 58.2(v)], as $Z\left(k C_{G}(F)\right)=\left(k C_{G}(F)\right)^{C_{G}(F)} \subset\left(k C_{G}(F)\right)^{E}$. We similarly denote by br $_{1}^{P}: k G \rightarrow$ $k C_{G}(P)$ the Brauer map defined analogously for any $p$-subgroup $P$ of $G$.

Cohomologically, the Brauer map $\operatorname{br}_{F}^{E}$ is the following composition of maps, where we identify $Z\left(k C_{G}(F)\right)$ with degree 0 Hochschild cohomology:

$\mathrm{H}^{0}\left(C_{G}(F), k C_{G}(F)\right) \stackrel{\operatorname{res}_{E}^{C_{G}(F)}}{\longrightarrow} \mathrm{H}^{0}\left(E, k C_{G}(F)\right) \rightarrow \overline{\mathrm{H}}^{0}\left(E, k C_{G}(F)\right) \cong \mathrm{H}^{0}\left(E, k C_{G}(E)\right)$. Indeed $\mathrm{H}^{0}\left(E, k C_{G}(E)\right) \cong\left(k C_{G}(E)\right)^{E}$, and the image of the above composition of maps lies in $k C_{G}(E)^{C_{G}(E)}=Z\left(k C_{G}(E)\right)$ since it factors through

$$
\mathrm{H}^{0}\left(C_{G}(E), k C_{G}(E)\right) .
$$

Since restriction and reducing modulo the ideal of proper transfers are algebra maps, we see that $\operatorname{br}_{F}^{E}: Z\left(k C_{G}(F)\right) \rightarrow Z\left(k C_{G}(E)\right)$ is also an algebra map.

In the case $F=1$, we may thus view the Brauer maps as degree 0 components of the map $\sigma$ defined in (2.2).

Let

$$
A^{\bullet}(E, F)=Z\left(k C_{G}(E)\right) \otimes \mathrm{H}^{\bullet}(F, k),
$$

and $A^{\bullet}(E), A^{\bullet}(F)$ be as defined in (2.6). Let

$$
\operatorname{Res}_{F}^{E}: A^{\bullet}(E) \rightarrow A^{\bullet}(E, F) \text { and } \operatorname{Br}_{F}^{E}: A^{\bullet}(F) \rightarrow A^{\bullet}(E, F)
$$

be defined by $\operatorname{Res} s_{F}^{E}=I d \otimes r e s_{F}^{E}$ and $\operatorname{Br}_{F}^{E}=\operatorname{br}_{F}^{E} \otimes I d$. Let

$$
\phi_{E, F}: \mathrm{H}^{\bullet}(G, k G) \rightarrow A^{\bullet}(E, F)
$$

be the composition of $\operatorname{res}_{F}^{G}$ and the following sequence of maps, where we note that the image of $\operatorname{res}_{F}^{G}$ is contained in the $C_{G}(E)$-invariant subalgebra:

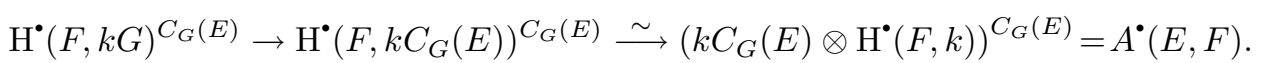

The first map above is induced by the Brauer map $b_{1}^{E}$, and the second is the isomorphism of Proposition 2.3(i).

Lemma 3.5. For elementary abelian p-subgroups $F<E$ of $G, \phi_{E, F}$ is an algebra homomorphism, and the following diagram commutes:

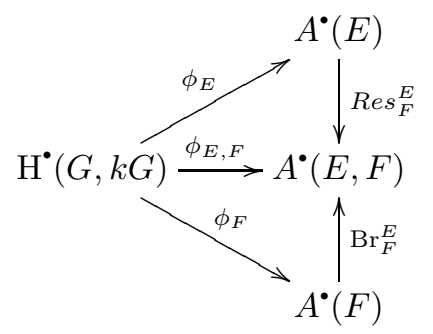

Proof. The map $\phi_{E}$ is essentially $r e s_{E}^{G}$ followed by the map induced by the Brauer map $\mathrm{br}_{1}^{E}$. From this it is clear that the top square commutes. The bottom square also commutes as $\phi_{E, F}$ is essentially $\phi_{F}$ followed by the map induced by the Brauer map $\operatorname{br}_{F}^{E}$. Finally, all the maps in the diagram other than $\phi_{E, F}$ are known to be algebra maps, and so commutativity of the diagram forces $\phi_{E, F}$ to be an algebra map as well. 
We may now restrict the domains of $\operatorname{Res}_{F}^{E}$ and $\operatorname{Br}_{F}^{E}$ to obtain maps $\operatorname{Res}_{F}^{E}$ : $\operatorname{Im}\left(\phi_{E}\right) \rightarrow \operatorname{Im}\left(\phi_{E, F}\right)$ and $\operatorname{Br}_{F}^{E}: \operatorname{Im}\left(\phi_{F}\right) \rightarrow \operatorname{Im}\left(\phi_{E, F}\right)$. We will next define auxiliary varieties $X_{G, E, F}$ and $Y_{G, E, F}$ that will be related to our previous varieties via these maps.

Definition 3.6. Let $Y_{G, E, F}=\max \left(A^{\bullet}(E, F)\right)$ and $X_{G, E, F}=\max \left(\operatorname{Im}\left(\phi_{E, F}\right)\right)$. By the same arguments as in the proof of Lemma 3.2 we have $Y_{G, E, F} \cong \bigcup_{B \in \mathfrak{B}_{E}} k^{\mathrm{rk}(F)}$, a disjoint union of affine spaces of dimension equal to the rank of $F$. The map $\phi_{E, F}$ induces a map of varieties $\phi_{E, F}^{*}: Y_{G, E, F} \rightarrow X_{G, E, F}$, Res $s_{F}^{E}$ induces $\left(\operatorname{Res}_{F}^{E}\right)^{*}$ : $X_{G, E, F} \rightarrow X_{G, E}$, and $\mathrm{Br}_{F}^{E}$ induces $\left(\operatorname{Br}_{F}^{E}\right)^{*}: X_{G, E, F} \rightarrow X_{G, F}$.

The following theorem gives the fundamental picture:

Theorem 3.7. If $F<E$ are elementary abelian p-subgroups of $G$, then the following diagram of varieties commutes:

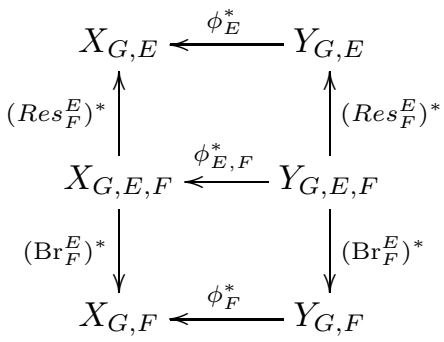

The maps $\phi_{E}^{*}, \phi_{F}^{*}, \phi_{E, F}^{*}$ are finite surjective maps. The maps $\left(\operatorname{Res}_{F}^{E}\right)^{*}$ are injective.

Proof. The commutativity of the diagram is a consequence of Lemma 3.5 and the comments in the paragraph following it. The injectivity of $\left(\operatorname{Res}_{F}^{E}\right)^{*}$ follows from the surjectivity of $\operatorname{Res}_{F}^{E}$ which in turn follows from the surjectivity of $\operatorname{res}_{F}^{E}: \mathrm{H}^{\bullet}(E, k) \rightarrow$ $\mathrm{H}^{\bullet}(F, k)$.

We have already seen that $\phi_{E}^{*}$ and $\phi_{F}^{*}$ are finite maps. Now $A^{\bullet}(E)$ is finitely generated over $\operatorname{Im}\left(\phi_{E}\right)$ and $\operatorname{Res}_{F}^{E}$ is surjective. Therefore $A^{\bullet}(E, F)$ is finitely generated over $\operatorname{Im}\left(\phi_{E, F}\right)$. It follows that $\phi_{E, F}$ is a finite map, and hence surjective as well.

By Theorem 3.7, we may view $X_{G, E, F}$ as the part of $X_{G, E}$ coming from $X_{G, F}$. Note that $Y_{G, E, F}$ has the same number of components as $Y_{G, E}$ in general, but their dimension is the same as that of $Y_{G, F}$. The map $\left(\operatorname{Res}_{F}^{E}\right)^{*}: Y_{G, E, F} \rightarrow Y_{G, E}$ may be defined componentwise for the blocks of $E$, the component associated to a block $B \in \mathfrak{B}_{E}$ being the map $\left(\operatorname{res}_{F}^{E}\right)^{*}: \max \left(\mathrm{H}^{*}(F, k)\right) \rightarrow \max \left(\mathrm{H}^{*}(E, k)\right)$. Thus we have a reasonably good understanding of the injective map $\left(\operatorname{Res}_{F}^{E}\right)^{*}$.

However, we will soon see that $\left(\operatorname{Br}_{F}^{E}\right)^{*}$ is neither injective nor surjective in general. It can happen that $F<E$ but $X_{G, F}$ does not inject into $X_{G, E}$, unlike the situation in the Quillen stratification of $\mathrm{H}^{\bullet}(G, k)$. We will now give a simple example to illustrate this.

Example 3.8. Let $G=\Sigma_{3}$ and $p=2$. Up to conjugacy, the only elementary abelian 2-subgroups are $E=\{1,(12)\}$ and 1 .

We have $A^{\bullet}(E)=k E \otimes \mathrm{H}^{\bullet}(E, k)$ and so $Y_{G, E}=k$. Similarly $A^{\bullet}(1)=Z(k G) \otimes k$ and so $Y_{G, 1}$ consists of two points (as $k G$ has two blocks). By the same reasoning, 
$Y_{G, E, 1}$ consists of exactly one point. It follows that $\left(\operatorname{Br}_{1}^{E}\right)^{*}: Y_{G, E, 1} \rightarrow Y_{G, 1}$ is not surjective.

In fact it will follow from Theorem 4.3 as $N_{G}(E)=E$, that $\phi_{1}^{*}, \phi_{E}^{*}, \phi_{E, 1}^{*}$ are injective and hence homeomorphisms onto their images. Thus we have $X_{G, E}=k$, $X_{G, 1}$ consists of two points and $X_{G, E} \cap X_{G, 1}$ consists of one point. Consequently, the Hochschild variety of $G$ is $X_{G}=X_{G, E} \cup X_{G, 1}=k \cup\{*\}$, where $*$ is an isolated point. Note that the trivial elementary abelian group contributed essentially to $X_{G}$ !

In terms of the block decomposition of $k G$, we have $X_{G}=\bigcup X_{B}$ where $X_{B}=$ $\max \left(\mathrm{HH}^{*}(B)\right)$, and $B$ ranges over the two blocks of $k G$. The dimension of $X_{B}$ is the $p$-rank of a defect group $P$ of $B$, that is, the rank of a maximal elementary abelian $p$-subgroup of $P$ (see Theorem 4.2 below or [15, Cor. 4.3(ii)]). Thus our picture $X_{G}=k \amalg\{*\}$ reflects the block structure of $k G$ : It has two blocks, one semisimple block (whose variety is $\{*\}$ ) and the principal block having variety $k$.

Now we work to better understand the map $\left(\operatorname{Br}_{F}^{E}\right)^{*}: Y_{G, E, F} \rightarrow Y_{G, F}$. The behaviour of this map is completely determined by $\left(\operatorname{br}_{F}^{E}\right)^{*}: \max \left(Z\left(k C_{G}(E)\right)\right) \rightarrow$ $\max \left(Z\left(k C_{G}(F)\right)\right)$, so we will study $\left(\mathrm{br}_{F}^{E}\right)^{*}$ first.

By [9, Prop. 56.16], $Z\left(k C_{G}(E)\right) / \operatorname{rad}\left(Z\left(k C_{G}(E)\right)\right) \cong \prod_{b \in \mathfrak{B}_{E}} k$, where the primitive central idempotent $e_{b}$ of $k C_{G}(E)$ projects to zero in all factors except the one corresponding to $B$, where it projects to 1 . Thus $\max \left(Z\left(k C_{G}(E)\right)\right.$ consists of a finite number of points, one for each block $b$ of $E$, and in this way we may identify $\max \left(Z\left(k C_{G}(E)\right)\right.$ with the set $\mathfrak{B}_{E}$ of blocks of $k C_{G}(E)$. Specifically, we associate to the block $b$ of $k C_{G}(E)$ the maximal ideal $M_{b}$ of $Z\left(k C_{G}(E)\right)$ that does not contain $e_{b}$.

The algebra map $\operatorname{br}_{F}^{E}: Z\left(k C_{G}(F)\right) \rightarrow Z\left(k C_{G}(E)\right)$ induces a map of varieties $\left(\operatorname{br}_{F}^{E}\right)^{*}: \max \left(Z\left(k C_{G}(E)\right)\right) \rightarrow \max \left(Z\left(k C_{G}(F)\right)\right)$ that may be viewed as a map from $\mathfrak{B}_{E}$ to $\mathfrak{B}_{F}$ by the above comments. In fact $\left(\mathrm{br}_{F}^{E}\right)^{*}$ is the block correspondence of Alperin and Broué: By [1, Lemma 3.6(1)], if $b$ is a block of $k C_{G}(E)$, there is a unique block $B$ of $k C_{G}(F)$ such that $\operatorname{br}_{F}^{E}\left(e_{B}\right) e_{b}=e_{b}$. The corresponding statement for the associated varieties is that $\left(\operatorname{br}_{F}^{E}\right)^{*}\left(M_{b}\right)$ does not contain $e_{B}$, and hence must be $M_{B}$. Conversely, since $\operatorname{br}_{F}^{E}$ is an algebra map from $Z\left(k C_{G}(F)\right)$ to $Z\left(k C_{G}(E)\right)$, the image $\operatorname{br}_{F}^{E}\left(e_{B}\right)$ is a central idempotent of $k C_{G}(E)$, and hence one of the following occurs:

(i) $\operatorname{br}_{F}^{E}\left(e_{B}\right)=0$, in which case $M_{B}$ is not in the image of $\left(\operatorname{br}_{F}^{E}\right)^{*}$, or

(ii) $\operatorname{br}_{F}^{E}\left(e_{B}\right)=\sum_{b \in S} e_{b}$ for some $S \subseteq \mathfrak{B}_{E}$, so that $\left(\operatorname{br}_{F}^{E}\right)^{*}\left(M_{b}\right)=M_{B}$ for all $b \in S$.

A consequence of (i) is that $\left(\mathrm{br}_{F}^{E}\right)^{*}$ may not be surjective, and a consequence of (ii) is that $\left(\mathrm{br}_{F}^{E}\right)^{*}$ may not be injective. Therefore $\left(\mathrm{Br}_{F}^{E}\right)^{*}$ is in general neither injective nor surjective. The following lemma summarizes implications for the relationship between the varieties $Y_{G, E, F}$ and $Y_{G, F}$.

Lemma 3.9. Let $F<E$ be elementary abelian p-subgroups of $G$. The Brauer map $\mathrm{Br}_{F}^{E}$ induces a map of varieties

$$
\left(\mathrm{Br}_{F}^{E}\right)^{*}: Y_{G, E, F}=\bigcup_{B \in \mathfrak{B}_{E}} k^{\mathrm{rk}(F)} \rightarrow Y_{G, F}=\bigcup_{B \in \mathfrak{B}_{F}} k^{\mathrm{rk}(F)}
$$

which identifies components according to the Alperin-Broué correspondence and is injective when restricted to any particular component. 
At this point, we have some understanding of the maps

$$
Y_{G, E} \stackrel{\left(\operatorname{Res}_{F}^{E}\right)^{*}}{\longleftarrow} Y_{G, E, F} \stackrel{\left(\operatorname{Br}_{F}^{E}\right)^{*}}{\longrightarrow} Y_{G, F}
$$

of Theorem 3.7. How well this carries over to the maps

$$
X_{G, E} \stackrel{\left(\operatorname{Res}_{F}^{E}\right)^{*}}{\longleftarrow} X_{G, E, F} \stackrel{\left(\mathrm{Br}_{F}^{E}\right)^{*}}{\longrightarrow} X_{G, F}
$$

depends on how far the finite surjective maps $\phi_{E}^{*}, \phi_{F}^{*}$ and $\phi_{E, F}^{*}$ are from being injective. We partially address this question in the next section, and use the auxiliary varieties $X_{G, E, F}$ to obtain a Quillen stratification of the Hochschild variety $X_{G}$. More precisely, we describe the stratification in terms of the blocks of $k G$.

\section{Quillen stratification and COnSEQuences}

In this section we will restrict our attention to the summands of $\mathrm{H}^{*}(G, k G)$ corresponding to the blocks of $k G$. By standard arguments (see for example [22, $\S 3])$,

$$
\operatorname{HH}^{*}(B)=\operatorname{Ext}_{B \otimes B^{o p}}^{*}(B, B) \cong \operatorname{Ext}_{k G \otimes(k G)^{o p}}^{*}(k G, B) \cong \operatorname{Ext}_{k G}^{*}(k, B),
$$

that is, $\operatorname{HH}^{*}(B) \cong \mathrm{H}^{*}(G, B)$, where $B$ is a $k G$-module via conjugation. Let $E$ be an elementary abelian $p$-subgroup of $G$. Recall that in degree 0, the map $\phi_{E}$ of (2.7) is essentially the Brauer map $\mathrm{br}_{1}^{E}$.

Let $B$ be a block of $k G$. We may apply $\phi_{E}$ to $\mathrm{H}^{*}(G, B)$ via the embedding $\mathrm{H}^{\bullet}(G, B) \hookrightarrow \mathrm{H}^{*}(G, k G)$, and we will denote this map by $\phi_{B, E}$. Similarly, let $\phi_{B, E, F}$ denote the map $\phi_{E, F}$ restricted to $\mathrm{H}^{*}(G, B)$. Now $\mathrm{H}^{\bullet}(G, B)=\mathrm{H}^{\bullet}\left(G, k G e_{B}\right)=$ $\mathrm{H}^{\bullet}(G, k G) e_{B}$ by identifying $e_{B}$ with an element of $\mathrm{H}^{0}(G, k G) \cong Z(k G)$. As $\phi_{E}$ is an algebra homomorphism,

$$
\begin{aligned}
\phi_{B, E}\left(\mathrm{H}^{\bullet}(G, B)\right) & =\phi_{E}\left(\mathrm{H}^{\bullet}(G, k G)\right) \phi_{E}\left(e_{B}\right) \\
& \subseteq\left(Z\left(k C_{G}(E)\right) \otimes \mathrm{H}^{*}(E, k)\right)\left(\mathrm{br}_{1}^{E}\left(e_{B}\right) \otimes 1\right) .
\end{aligned}
$$

Thus we have

$$
\operatorname{Im}\left(\phi_{B, E}\right) \subseteq \operatorname{br}_{1}^{E}\left(e_{B}\right) Z\left(k C_{G}(E)\right) \otimes \mathrm{H}^{\bullet}(E, k),
$$

and further the image is an $N_{G}(E)$-invariant subalgebra.

Let

$$
\begin{aligned}
A_{B}^{\bullet}(E) & =\operatorname{br}_{1}^{E}\left(e_{B}\right) Z\left(k C_{G}(E)\right) \otimes \mathrm{H}^{\bullet}(E, k), \\
\text { and } A_{B}^{\cdot}(E, F) & =\operatorname{br}_{1}^{E}\left(e_{B}\right) Z\left(k C_{G}(E)\right) \otimes \mathrm{H}^{\bullet}(F, k) .
\end{aligned}
$$

We next define the corresponding varieties.

Definition 4.1. The Hochschild variety of the block $B$ of $k G$ is

$$
X_{B}=\max \left(\mathrm{H}^{\bullet}(G, B)\right) \text {. }
$$

Let $Y_{B, E}=\max \left(A_{B}^{\cdot}(E)\right)$ and $Y_{B, E, F}=\max \left(A_{B}^{\cdot}(E, F)\right)$. By restricting the domains of the maps (3.4), we have $\operatorname{Res}_{F}^{E}: A_{B}^{*}(E) \rightarrow A_{B}^{\cdot}(E, F)$ and $\operatorname{Br}_{F}^{E}: A_{B}^{*}(F) \rightarrow$ $A_{B}^{\bullet}(E, F)$. These induce maps on varieties

$$
Y_{B, F} \stackrel{\left(\mathrm{Br}_{F}^{E}\right)^{*}}{\longleftarrow} Y_{B, E, F} \stackrel{\left(\operatorname{Res}_{F}^{E}\right)^{*}}{\hookrightarrow} Y_{B, E} .
$$

Let

$$
Y_{B, E}^{+}=Y_{B, E}-\bigcup_{F<E}\left(\operatorname{Res}_{F}^{E}\right)^{*}\left(Y_{B, E, F}\right) .
$$


Similarly, let $X_{B, E}=\max \left(\operatorname{Im}\left(\phi_{B, E}\right)\right), X_{B, E, F}=\max \left(\operatorname{Im}\left(\phi_{B, E, F}\right)\right)$, and

$$
X_{B, E}^{+}=X_{B, E}-\bigcup_{F<E}\left(\operatorname{Res}_{F}^{E}\right)^{*}\left(X_{B, E, F}\right) .
$$

Note that by Theorem 3.7, $X_{B, E}^{+}=\phi_{B, E}^{*}\left(Y_{B, E}^{+}\right)$.

The above observations and those at the end of the last section allow us to describe the varieties $X_{B, E}$ in terms of the defect groups of $B$ : These are the maximal $p$-subgroups $P$ of $G$ such that $\operatorname{br}_{1}^{P}\left(e_{B}\right) \neq 0$ [1, (2.6)]. They are all conjugate in $G$, and if $P$ is any $p$-subgroup, $\operatorname{br}_{1}^{P}\left(e_{B}\right) \neq 0$ if and only if $P$ is contained in a defect group of $B$ 11, (2.6)]. Thus $X_{B, E}$ is nonempty if, and only if, $E$ is contained in a defect group of $B$. The following theorem is the first part of the Quillen stratification, giving a more precise description of the Hochschild variety $X_{B}$ of $B$.

Theorem 4.2. Let $B$ be a block of $G$ and $P$ a defect group of $B$. Then

$$
X_{B}=\bigcup_{E} X_{B, E}^{+}
$$

a disjoint union of subvarieties, taken over a set of elementary abelian p-subgroups $E$ of $P$, one from each $G$-conjugacy class of elementary abelian p-subgroups of $G$ for which at least one member is contained in $P$. Further there are finite surjective maps $Y_{B, E}^{+} \rightarrow X_{B, E}^{+}$, so that $\operatorname{dim}\left(X_{B, E}^{+}\right)=\operatorname{dim}\left(Y_{B, E}^{+}\right)=\operatorname{rk}(E)$, and $\operatorname{dim}\left(X_{B}\right)=\operatorname{rk}(P)$.

We remark that $\operatorname{dim}\left(X_{B}\right)$ was already known to be equal to $\operatorname{rk}(P)$, as follows from [15, Cor. 4.3(ii)].

Proof. We use standard methods, such as in [7, §5.6]. For each $E$, let

$$
\sigma_{E}=\prod_{0 \neq \zeta \in \mathrm{H}^{1}\left(E, \mathbb{F}_{p}\right)} \beta(\zeta),
$$

where $\beta$ is the Bockstein homomorphism, so that $\sigma_{E} \in \mathrm{H}^{\bullet}(E, k)$. By definition of the map $\operatorname{Res}_{F}^{E}$, we now have $Y_{B, E}^{+}=\max \left(A_{B}^{*}(E)\left[\operatorname{br}_{1}^{E}\left(e_{B}\right) \otimes \sigma_{E}^{-1}\right]\right)$. Note that $Y_{B, E}^{+}$is empty if $\operatorname{br}_{1}^{E}\left(e_{B}\right)=0$, which is the case if no $G$-conjugate of $E$ lies in $P$.

By [7, Lemma 5.6.2], for each elementary abelian $p$-subgroup $E$ of $G$, there is an element $\rho_{E} \in \mathrm{H}^{*}(G, k)$ such that $r e s_{E}^{G}\left(\rho_{E}\right)=\left(\sigma_{E}\right)^{p^{a}}$ for some $a$. Further, if $F$ is an elementary abelian $p$-subgroup that is not contained in a conjugate of $E$, then $\operatorname{res}_{E}^{G}\left(\rho_{F}\right)=0$. Let $\psi_{B}: \mathrm{H}^{*}(G, k) \rightarrow \mathrm{H}^{*}(G, B)$ be the map induced by the inclusion $k \hookrightarrow B$ that sends 1 to $e_{B}$. We have $\phi_{B, E}\left(\psi_{B}\left(\rho_{F}\right)\right)=\operatorname{br}_{1}^{E}\left(e_{B}\right) \otimes \operatorname{res}_{E}^{G}\left(\rho_{F}\right)$ for all elementary abelian $p$-subgroups $F \leq E$ of $G$, which is nonzero if and only if $E$ is contained in a conjugate of $P$ and $F$ is contained in a conjugate of $E$. It follows from these observations that

$$
\begin{aligned}
X_{B, E}^{+} & =\max \left(\phi_{B, E}\left(\mathrm{H}^{\bullet}(G, B)\left[\psi_{B}\left(\rho_{E}\right)^{-1}\right]\right)\right) \\
& =\max \left(\operatorname{Im}\left(\phi_{B, E}\right)\left[\left(\operatorname{br}_{1}^{E}\left(e_{B}\right) \otimes \operatorname{res}_{E}^{G}\left(\rho_{E}\right)\right)^{-1}\right]\right) .
\end{aligned}
$$

Now $\operatorname{Im}\left(\phi_{B, E}\right)$ is an $\mathrm{H}^{\bullet}(G, B)$-module via $\phi_{B, E}$, and $\psi_{B}\left(\rho_{F}\right) \in \mathrm{H}^{\bullet}(G, B)$ acts on $\operatorname{Im}\left(\phi_{B, E}\right)$ as $\operatorname{br}_{1}^{E}\left(e_{B}\right) \otimes \operatorname{res}_{E}^{G}\left(\rho_{F}\right)$, which is zero if no $G$-conjugate of $F$ is contained in $E$. Thus the $X_{B, E}^{+}$are disjoint since given a pair of elementary abelian $p$-subgroups $E$ and $F$ which are not $G$-conjugate, at least one of them does not contain the other up to $G$-conjugacy. 
Also note that the only maximal ideals $M$ removed from $X_{B, E}$ to form $X_{B, E}^{+}$are those which contain $\operatorname{br}_{1}^{E}\left(e_{B}\right) \otimes \operatorname{res}_{E}^{G}\left(\rho_{E}\right)$ or equivalently $\operatorname{br}_{1}^{E}\left(e_{B}\right) \otimes\left(\sigma_{E}\right)^{p^{a}}$. Pulling $M$ back to $\mathrm{H}^{*}(G, B)$, we have an ideal containing $\operatorname{Ker}\left(\phi_{B, E}\right)$ and $\psi_{B}\left(\rho_{E}\right)$. This ideal is in the image $\left(\operatorname{Res}_{F}^{E}\right)^{*}\left(X_{B, E, F}\right)$ for some $F<E$ and hence the ideal $M$ contains $\operatorname{Ker}\left(\phi_{B, E, F}\right)$ and $\operatorname{Ker}\left(\phi_{B, F}\right)$. Thus $M$ will be in $X_{B, F}$ for some $F<E$. It follows that the union of $X_{B, E}^{+}$, over all elementary abelian $p$-subgroups $E$, is still $X_{B}$

We now give a more complete description of the varieties $X_{B, E}^{+}$in a special case. Let $W_{G}(E)=N_{G}(E) / C_{G}(E)$, the Weyl group of $E$.

Theorem 4.3. Let $B$ be a block of $G$ and $E$ an elementary abelian p-subgroup of $G$ such that $\operatorname{br}_{1}^{E}\left(e_{B}\right)=e_{b}$ for some block $b$ of $C_{G}(E)$. Then $\phi_{B, E}^{*}$ induces an inseparable isogeny $Y_{B, E}^{+} / W_{G}(E) \rightarrow X_{B, E}^{+}$.

The relationship between $Y_{B, E}^{+}$and $X_{B, E}^{+}$in general appears to be more subtle, as the maps $\phi_{B, E}$ may be more complicated.

Proof. Under the hypothesis, $A_{B}^{\bullet}(E)=Z(b) \otimes \mathrm{H}^{\bullet}(E, k)$. Let $\gamma_{B, E}: \mathrm{H}^{\bullet}(G, k) \rightarrow$ $A_{B}^{\bullet}(E)$ be the composition

$$
\mathrm{H}^{\bullet}(G, k) \stackrel{\psi_{B}}{\longrightarrow} \mathrm{H}^{\bullet}(G, B) \stackrel{\phi_{B, E}}{\longrightarrow} A_{B}^{\bullet}(E),
$$

where as above $\psi_{B}$ is induced by the inclusion $k \hookrightarrow B$ that sends 1 to $e_{B}$. Note that $\gamma_{B, E}$ sends $\zeta \in \mathrm{H}^{\bullet}(G, k)$ to $\operatorname{br}_{1}^{E}\left(e_{B}\right) \otimes \operatorname{res}_{E}^{G}(\zeta)$. As elements of $\mathrm{H}^{\bullet}(G, k)$ and of $\mathrm{H}^{*}(G, B)$ are invariant under conjugation by elements of $G$, we have the following containments:

$$
\operatorname{Im}\left(\gamma_{B, E}\right) \subseteq \operatorname{Im}\left(\phi_{B, E}\right) \subseteq A_{B}^{*}(E)^{W_{G}(E)} .
$$

Every element in $A_{B}^{\bullet}(E)^{W_{G}(E)}$ may be expressed as $e_{b} \otimes \zeta$, for some $\zeta \in \mathrm{H}^{\bullet}(E, k)^{W_{G}(E)}$, plus an element in the radical. (This follows from writing $x \in A_{B}^{\cdot}(E)^{W_{G}(E)}$ as $x=\sum_{i}\left(c_{i} e_{b}+r_{i}\right) \otimes \zeta_{i}$ where $c_{i} \in k$ and $r_{i} \in \operatorname{rad}(Z(b))$.)

By [7, Lemma 5.6.2], there is a nonnegative integer $a$ such that $\zeta^{p^{a}}=\operatorname{res}_{E}^{G}\left(\rho_{E}^{-1} \zeta^{\prime}\right)$ for some $\zeta^{\prime} \in \mathrm{H}^{*}(G, k)$ and $\rho_{E} \in \mathrm{H}^{*}(G, k)$ as before with $\operatorname{res}_{E}^{G}\left(\rho_{E}\right)=\left(\sigma_{E}\right)^{p^{a}}$. Therefore $\left(e_{b} \otimes \zeta\right)^{p^{a}}=e_{b} \otimes \zeta^{p^{a}}=e_{b} \otimes \operatorname{res}_{E}^{G}\left(\rho_{E}^{-1} \zeta^{\prime}\right) \in \operatorname{Im}\left(\gamma_{B, E}\right)$, where we have extended $\gamma_{B, E}$ to a map from $\mathrm{H}^{\bullet}(G, k)\left[\rho_{E}^{-1}\right]$ to $\left(A_{B}^{\cdot}(E)\left[e_{b} \otimes \sigma_{E}^{-1}\right]\right)^{W_{G}(E)}$. Considering the sequence of subalgebras (4.4), this shows that $\left(e_{b} \otimes \zeta\right)^{p^{a}}$ is also contained in $\phi_{B, E}\left(\mathrm{H}^{\bullet}(G, B)\left[\psi_{B}\left(\rho_{E}\right)^{-1}\right]\right)$. Thus

$$
\left(\left(A_{B}^{\cdot}(E)\left[e_{b} \otimes \sigma_{E}^{-1}\right]\right)^{W_{G}(E)}\right)^{p^{a}} \subseteq \operatorname{Im}\left(\phi_{B, E}\right)\left[e_{b} \otimes \sigma_{E}^{-1}\right] \subseteq\left(A_{B}^{\bullet}(E)\left[e_{b} \otimes \sigma_{E}^{-1}\right]\right)^{W_{G}(E)} .
$$

Hence $\phi_{B, E}$ induces an inseparable isogeny

$$
\max \left(\left(A_{B}^{\bullet}(E)\left[e_{b} \otimes \sigma_{E}^{-1}\right]\right)^{W_{G}(E)}\right)=Y_{B, E}^{+} / W_{G}(E) \rightarrow X_{B, E}^{+} .
$$

The principal block $B_{0}$ of $G$ satisfies the hypotheses of Theorem 4.3 for each elementary abelian $p$-subgroup $E$ of $G$, that is, the defect groups of $B_{0}$ are the Sylow $p$-subgroups, and $\operatorname{br}_{1}^{E}\left(e_{B_{0}}\right)=e_{b_{0}}$ for every elementary abelian $p$-subgroup $E$ of $G$. We are therefore led to the following corollary, which proves a weak form of the conjecture [23, Conj. 1]. 
Corollary 4.5. Let $B_{0}$ be the principal block of $k G$. Then $\max \left(\mathrm{HH}^{*}\left(B_{0}\right)\right)$ is homeomorphic to $\max \left(\mathrm{H}^{*}(G, k)\right)$.

Proof. First note that all elementary abelian $p$-subgroups of $G$ are $G$-conjugate to a subgroup of the Sylow $p$-subgroup $P$, the defect group of $B_{0}$.

By the proof of Theorem 4.3, the maps $\phi_{B_{0}, E}$ together give a homomorphism $\phi_{B_{0}}: \mathrm{H}^{\bullet}\left(G, B_{0}\right) \rightarrow \lim A_{B_{0}}^{\cdot}(E)$, where this inverse limit is over the category of elementary abelian $p$-subgroups of $G$ with morphisms given by inclusions and $G$ conjugations. Note that since $\operatorname{br}_{1}^{E}\left(e_{B_{0}}\right)=e_{b_{0}}$, there is no ambiguity in defining the gluing maps in this inverse limit.

The proofs of Theorems 4.2 and 4.3 show that modulo radicals, $\phi_{B_{0}}$ induces an inseparable isogeny. However since $\operatorname{br}_{1}^{E}\left(e_{B_{0}}\right)=e_{b_{0}}$ for every elementary abelian $p$ subgroup $E$, we see that modulo radicals, $\lim A_{B_{0}}(E)$ is isomorphic to $\lim H^{\bullet}(E, k)$. Quillen has shown [7, Thm. 5.6.3] that there is an inseparable isogeny between this latter inverse limit and $\mathrm{H}^{*}(G, k)$. It follows that the varieties for $\mathrm{H}^{*}(G, k)$ and $\mathrm{H}^{*}\left(G, B_{0}\right)$ are homeomorphic.

More generally, Theorem 4.3 applies to a block of principal type, that is, a block $B$ such that for every $p$-subgroup $P$ of $G, \operatorname{br}_{1}^{P}\left(e_{B}\right)$ is either 0 or a unique block idempotent $e_{b}$. For such a block $B$, Theorem 4.3 implies that the Hochschild variety $X_{B}$ is homeomorphic to the subvariety of $\max \left(\mathrm{H}^{*}(G, k)\right)$ obtained by removing those pieces in the Quillen stratification corresponding to elementary abelian $p$-subgroups not contained in a defect group of $B$.

In the remainder of this section, we will speculate on related potential consequences of Theorems 4.2 and 4.3 Let $\left(P, B_{P}\right)$ be a Sylow $B$-subpair of $G$, unique up to conjugacy [1, so that $P$ is a defect group of $B$ and $B_{P}$ is a block of $k C_{G}(P)$. If $R$ is a subgroup of $P$, there is a unique block $B_{R}$ of $k C_{G}(R)$ such that $\left(R, B_{R}\right) \leq\left(P, B_{P}\right)$, where the partial order on subpairs is defined in [1]. Let $N_{G}\left(B_{R}\right)$ be the subgroup of $N_{G}(R)$ fixing $B_{R}$ setwise, under conjugation.

Definition 4.6 (Linckelmann [14, 15]). Let $B$ be a block of $k G$ with defect group $P$. The block cohomology ring of $B$ is the subring $\operatorname{LH}^{*}(B)$ of $\mathrm{H}^{*}(P, k)$ consisting of all $\zeta \in \mathrm{H}^{*}(P, k)$ satisfying

$$
{ }^{g} \operatorname{res}_{R}^{P}(\zeta)=\operatorname{res}_{R}^{P}(\zeta)
$$

for all subgroups $R$ of $P$, and all $g \in N_{G}\left(B_{R}\right)$.

If $B=B_{0}$ is the principal block, then $\operatorname{LH}^{*}\left(B_{0}\right) \cong \mathrm{H}^{*}(G, k)$. Thus Corollary 4.5 shows that $\max \left(\mathrm{HH}^{*}\left(B_{0}\right)\right)$ is homeomorphic to $\max \left(\mathrm{LH}^{*}\left(B_{0}\right)\right)$. We do not know whether the generalization of this statement to all blocks is true, so we raise it as a question.

Question 4.7. Let $B$ a block of $k G$. Are $\max \left(\mathrm{HH}^{*}(B)\right)$ and $\max \left(\mathrm{LH}^{*}(B)\right)$ homeomorphic?

In fact, the cohomology rings $\mathrm{HH}^{*}(B)$ and $\mathrm{LH}^{*}(B)$ are isomorphic, modulo their radicals, in many known cases as shown in [17, and the question was raised in that paper whether this is true in general. We do not know of a counterexample to either statement. It is possible that a detailed comparison of our stratification of the Hochschild variety $\max \left(\mathrm{HH}^{*}(B)\right)$ of a block $B$ with Linckelmann's stratification of the block variety $\max \left(\mathrm{LH}^{*}(B)\right)$ [16] would yield further information, potentially providing an answer to this question. Another approach, suggested by the referee, 
would begin with proving a generalization of Theorem 4.3, where $W_{G}(E)$ is replaced by $W_{G}(E, b)$. (Here $W_{G}(E, b)$ is the normalizer modulo centralizer of a pair $\left(E, e_{b}\right)$ for some block $e_{b}$ of $C_{G}(E)$ occurring in $\operatorname{br}_{1}^{E}\left(e_{B}\right)$.)

In Linckelmann's paper [16], he also deals more generally with the support varieties of modules over a block $B$, defined via the block cohomology $\operatorname{LH}^{*}(B)$. An analogous theory defined via the Hochschild cohomology $\operatorname{HH}^{*}(B)$ was developed in the unpublished work of Siegel [21], some of which has now been done in greater generality by Snashall and Solberg 24] for modules over Artinian algebras. The techniques we have developed in this paper should lead to a Quillen stratification of these support varieties of modules over $B$, defined via the Hochschild cohomology $\mathrm{HH}^{*}(B)$, but we do not pursue this here.

\section{ACKNOWLEDGMENT}

The second author thanks M. Linckelmann for some very helpful discussions.

\section{Appendix: Hochschild COHOMOLOGY AND ELEMENTARY ABELIAN SUBGROUPS}

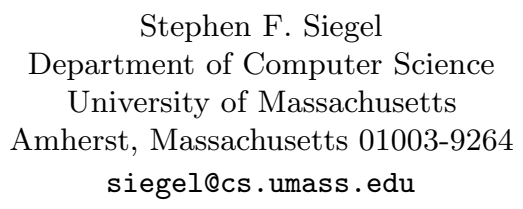

Main results. Throughout this section, $G$ is a finite group and $k$ is a (not necessarily algebraically closed) field of prime characteristic $p$. All $k$-algebras are assumed to have units.

By a $G$-equivariant $k$-algebra we mean a $k$-algebra $A$ on which $G$ acts as algebra automorphisms. A map of $G$-equivariant $k$-algebras is an algebra homomorphism which is also a $k G$-homomorphism. By a $G$-equivariant $A$-module we mean an $A$-module $M$ such that the structure map

$$
\mu: A \otimes M \rightarrow M
$$

is a $k G$-homomorphism, i.e., $g(a x)=(g a)(g x)$ for all $g \in G, a \in A$, and $x \in M$. A map of $G$-equivariant $A$-modules is an $A$-homomorphism which is also a $k G$ homomorphism.

In this setting, the cup product gives $\mathrm{H}^{*}(G, A)$ the structure of a graded $k$ algebra, and $\mathrm{H}^{*}(G, M)$ becomes an $\mathrm{H}^{*}(G, A)$-module.

The main result is the following key lemma.

Lemma 5.1. Let $A$ be a $G$-equivariant k-algebra, and $\zeta \in \mathrm{H}^{*}(G, A)$. Let $M$ be a $G$-equivariant $A$-module. Suppose that, for each elementary abelian p-subgroup $E$ of $G$, some power of $\operatorname{res}_{E}^{G}(\zeta) \in \mathrm{H}^{*}(E, A)$ annihilates $\mathrm{H}^{*}(E, M)$. Then some power of $\zeta$ annihilates $\mathrm{H}^{*}(G, M)$.

Before proving Lemma 5.1 , we look at several consequences. It will be seen that the lemma allows one to easily obtain several of the well-known results in ordinary group cohomology, as well as analogous results for Hochschild cohomology, in a unified way.

The main application is in the following setting. Suppose $N$ is a $G$-equivariant $k$-algebra, and that there is a map of $G$-equivariant algebras $\alpha: A \rightarrow N$. Then we 
may consider $N$ as a $G$-equivariant $A$-module via $\mu(a \otimes x)=\alpha(a) x$. In this case we can say something in both directions.

Theorem 5.2. Let $A$ and $\zeta$ be as in Lemma 5.1, and $\alpha: A \rightarrow N$ as above. Then some power of $\zeta$ annihilates $\mathrm{H}^{*}(G, N)$ if, and only if, for each elementary abelian p-subgroup $E$ of $G$, some power of $\operatorname{res}_{E}^{G}(\zeta)$ annihilates $\mathrm{H}^{*}(E, N)$.

Proof. Since we are dealing with algebras with units, $\zeta^{n}$ annihilates $\mathrm{H}^{*}(E, N)$ if, and only if, it annihilates $1 \in \mathrm{H}^{0}(E, N)$. Assuming $\zeta^{n}$ annihilates $\mathrm{H}^{*}(G, N)$, we have

$$
\operatorname{res}_{E}^{G}\left(\zeta^{n}\right) \cdot 1=\operatorname{res}_{E}^{G}\left(\zeta^{n}\right) \cdot \operatorname{res}_{E}^{G}(1)=\operatorname{res}_{E}^{G}\left(\zeta^{n} \cdot 1\right)=0 .
$$

So $\zeta^{n}$ annihilates $H^{*}(E, N)$. The other direction follows from the lemma.

The special case of Theorem 5.2 in which $N=A$ and $\alpha=1$ yields the following:

Corollary 5.3. Let $A$ be a $G$-equivariant k-algebra, and $\zeta \in \mathrm{H}^{*}(G, A)$. Then $\zeta$ is nilpotent if, and only if, $\operatorname{res}_{E}^{G}(\zeta)$ is nilpotent for every elementary abelian subgroup $E$ of $G$.

The case $A=k$ is well known and is a key step in the ordinary Quillen stratification for $\mathrm{H}^{*}(G, k)$. Another case is where $M$ is a $k G$-module and $N=\operatorname{End}_{k}(M)$. In that case there is a natural isomorphism

$$
\mathrm{H}^{*}(G, N) \cong \operatorname{Ext}_{k G}^{*}(M, M) \text {. }
$$

So Corollary 5.3 yields the well-known result that an element of $\operatorname{Ext}_{k G}^{*}(M, M)$ is nilpotent if and only if its restriction to $E$ is nilpotent for every elementary abelian E.

We may also take $A=k$ and $N=\operatorname{End}_{k}(M)$ and obtain from Theorem 5.2 the weaker well-known result that for $\zeta \in \mathrm{H}^{*}(G, k)$, some power of $\zeta$ annihilates $\operatorname{Ext}_{k G}^{*}(M, M)$ if and only if for all $E$, some power of $\operatorname{res}_{E}^{G}(\zeta)$ annihilates $\operatorname{Ext}_{k E}^{*}(M, M)$.

To see how this applies to Hochschild cohomology, recall that $\operatorname{HH}^{*}(k G)$, the Hochschild cohomology of $k G$, may be identified with $\mathrm{H}^{*}(G, k G)$, where $k G$ is considered a module under conjugation. In this case, Corollary 5.3 yields

Corollary 5.4. Let $\zeta \in \mathrm{HH}^{*}(k G)$. Then $\zeta$ is nilpotent if, and only if, its restriction to $\mathrm{H}^{*}(E, k G)$ is nilpotent for every elementary abelian p-subgroup $E$ of $G$.

We also get a module version for Hochschild cohomology, as follows. Let $A=k G$, let $M$ be a $k G$-module and let $N=\operatorname{End}_{k}(M)$. We let $G$ act on $A$ by conjugation, and on $N$ by $(g f)(x)=g f\left(g^{-1} x\right)$. Then the map $A \rightarrow N$ which takes $g$ to the map $(x \mapsto g x)$, is $G$-equivariant. Hence Theorem 5.2 says the following:

Corollary 5.5. Let $M$ be a $k G$-module and $\zeta \in \mathrm{HH}^{*}(k G)$. Then some power of $\zeta$ annihilates $\operatorname{Ext}_{k G}^{*}(M, M)$ if, and only if, for each elementary abelian p-subgroup $E$ of $G$, some power of $\operatorname{res}_{E}^{G}(\zeta)$ annihilates $\operatorname{Ext}_{k E}^{*}(M, M)$.

Corollary 5.5 has an interpretation in terms of varieties for modules. Consider $X_{G}$, the (prime or maximal ideal) spectrum of $\mathrm{HH}^{*}(k G)$. Let $X_{G}(M)=V(I)$, the subvariety of $X_{G}$ consisting of the (prime or maximal) ideals containing I, where $I$ is the radical of the annihilator in $\operatorname{HH}^{*}(k G)$ of $\operatorname{Ext}_{k G}^{*}(M, M)$; we call $X_{G}(M)$ the Hochschild support variety for $M$ (cf. 24]). More generally, for any subgroup $H$ of $G$, let $X_{G, H}(M)=V(J)$, where $J$ is the radical of the kernel of the composite

$$
\mathrm{H}^{*}(G, k G) \rightarrow \operatorname{Ext}_{k G}^{*}(M, M) \rightarrow \operatorname{Ext}_{k H}^{*}(M, M),
$$


in which the second map is a restriction. We may think of $X_{G, H}(M)$ as the part of $X_{G}(M)$ coming from $H$. Now Corollary 5.5 is easily seen to be equivalent to the following:

Corollary 5.6. Let $M$ be a $k G$-module. Then

$$
X_{G}(M)=\bigcup_{E} X_{G, E}(M)
$$

where the union is taken over all elementary abelian p-subgroups $E$ of $G$.

The analogous result for ordinary cohomology is the well-known Alperin-Evens Theorem (3]).

Results needed for proof. The proof of Lemma 5.1 follows the one for the usual case ( $k$ in place of $A$ ) given in [11, Cor. 8.3.4], which uses Serre's Theorem on the product of Bocksteins, and the module version of the Quillen-Venkov Lemma. We just have to make a few adjustments along the way.

The first ingredient is this commutativity lemma.

Lemma 5.7. Let $G$ be a finite group, $k$ a field, $A$ a G-equivariant $k$-algebra, and $\eta: k \rightarrow A$ the map sending $\lambda$ to $\lambda 1$. Then the image of the algebra homomorphism

$$
\eta^{*}: \mathrm{H}^{*}(G, k) \rightarrow \mathrm{H}^{*}(G, A)
$$

is contained in the graded-center of $\mathrm{H}^{*}(G, A)$.

Proof. There is a commutative diagram

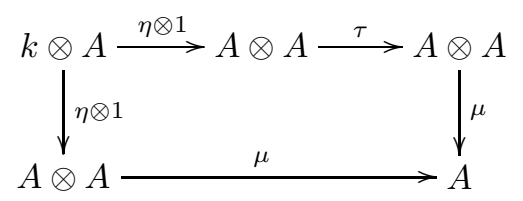

where $\tau(x \otimes y)=y \otimes x$ and $\mu(x \otimes y)=x y$. The lemma then follows from the definition of the cup product.

Next, a definition. Suppose $P$ is a $p$-group and $K$ is a maximal subgroup of $P$. Then $K$ is normal and $P / K$ is cyclic of order $p$, so $\mathrm{H}^{1}(P / K, k) \cong k$. Let $\alpha$ be a nonzero element of $\mathrm{H}^{1}(P / K, k)$ and define

$$
\beta_{K}=\beta\left(\inf _{P / K}^{P}(\alpha)\right) \in H^{2}(P, k),
$$

where $\beta: \mathrm{H}^{s}(P, k) \rightarrow \mathrm{H}^{s+1}(P, k)(s \geq 0)$ is the Bockstein map and $\inf _{P / K}^{P}$ is the inflation map [11, $\S \S 1.1,3.3]$. Of course $\beta_{K}$ is only well defined up to nonzero scalar multiple, but the particular choice does not matter for what follows.

Theorem 5.8 (Serre, 20). Let $P$ be a p-group. Then $P$ is not elementary abelian if, and only if, there exist maximal subgroups $K_{1}, \ldots, K_{r}$ of $P$ such that

$$
\beta_{K_{1}} \ldots \beta_{K_{r}}=0
$$

in $H^{*}(P, k)$.

For a simple proof, see [11, Thm. 6.4.1]. The statement there differs only in that it is stated in terms of integral cohomology, but as explained in the proof of [11, Cor. 6.4.2], this implies the mod- $p$ version. 
The other ingredient we need is a lemma of Alperin and Evens ([2, Lemma 4.1]), which generalizes to arbitrary modules the Quillen-Venkov Lemma ([19]):

Lemma 5.9 (Quillen-Venkov, Alperin-Evens). Let $P$ be a p-group, $K$ a maximal subgroup of $P$, and $M$ a $k P$-module. Then the filtration of $\mathrm{H}^{*}(P, M)$ associated to the Lyndon-Hochschild-Serre spectral sequence

$$
\mathrm{H}^{*}\left(P / K, \mathrm{H}^{*}(K, M)\right) \Rightarrow \mathrm{H}^{*}(P, M)
$$

satisfies

$$
F^{2} \mathrm{H}^{*}(P, M)=\beta_{K} \mathrm{H}^{*}(P, M) .
$$

Proof of Lemma 5.1, We assume that for each elementary abelian $p$-subgroup $E$ of $G$, some power of $\operatorname{res}_{E}^{G}(\zeta)$ annihilates $\mathrm{H}^{*}(E, M)$, and we will show that some power of $\zeta$ annihilates $\mathrm{H}^{*}(G, M)$.

Let $S$ be a Sylow $p$-subgroup of $G$. It suffices to show that some power of $\operatorname{res}_{S}^{G}(\zeta)$ annihilates $\mathrm{H}^{*}(S, M)$, since restriction from $\mathrm{H}^{*}(G, M)$ to $\mathrm{H}^{*}(S, M)$ is injective [6, Cor. 3.6.18]. We will do this by showing that some power of $\operatorname{res}_{P}^{G}(\zeta)$ annihilates $\mathrm{H}^{*}(P, M)$ for every subgroup $P$ of $S$, by induction on $|P|$.

If $|P|=1$ the result is clear. So suppose $|P|>1$ and we have established the result for every proper subgroup of $P$. If $P$ is elementary abelian, we are done by hypothesis. If not, then let $K_{1}, \ldots, K_{r}$ be maximal subgroups of $P$ as in Theorem 5.8, and let $\beta_{i}=\beta_{K_{i}}$.

Let $K=K_{1}$. For any $k P$-module $U$, let $E(U)$ denote the Lyndon-HochschildSerre spectral sequence

$$
\mathrm{H}^{*}\left(P / K, \mathrm{H}^{*}(K, U)\right) \Rightarrow \mathrm{H}^{*}(P, U) .
$$

Now $E(M)$ is a module over $E(A)$ and over $E(k)$, and the commutative diagram

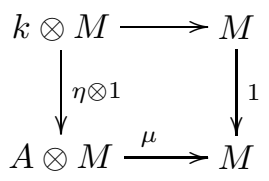

implies that the following diagram commutes:

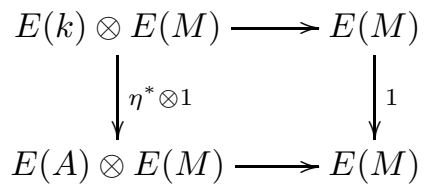

Let $\chi=r e s_{P}^{G}(\zeta)$. By induction, for some $m=m_{1}, \xi=\operatorname{res}_{K}^{P}\left(\chi^{m}\right) \in E_{2}^{0, *}(A)$ annihilates $\mathrm{H}^{*}(K, M)$, and therefore $E_{2}(M)$. Since $\xi$ is in the image of $\operatorname{res}_{K}^{P}, \xi$ lives to $E_{\infty}(A)$, and so it annihilates $E_{\infty}(M)$ as well. This means that

$$
\chi^{m} F^{i} \mathrm{H}^{*}(P, M) \subseteq F^{i+1} \mathrm{H}^{*}(P, M)
$$

for all $i \geq 0$. In particular,

$$
\chi^{2 m_{1}} \mathrm{H}^{*}(P, M) \subseteq F^{2} \mathrm{H}^{*}(P, M)=\beta_{1} \mathrm{H}^{*}(P, M),
$$

by Lemma 5.9 ,

Applying the same reasoning to $K=K_{2}$, there is some $m_{2}$ such that

$$
\chi^{2 m_{2}} \mathrm{H}^{*}(P, M) \subseteq \beta_{2} \mathrm{H}^{*}(P, M) .
$$


So

$$
\begin{aligned}
\chi^{2 m_{1}+2 m_{2}} \mathrm{H}^{*}(P, M) & \subseteq \chi^{2 m_{2}}\left(\beta_{1} \mathrm{H}^{*}(P, M)\right) \\
& =\left(\chi^{2 m_{2}} \eta^{*}\left(\beta_{1}\right)\right) \mathrm{H}^{*}(P, M) \\
& =\left(\eta^{*}\left(\beta_{1}\right) \chi^{2 m_{2}}\right) \mathrm{H}^{*}(P, M)
\end{aligned}
$$

since, by Lemma 5.7, $\eta^{*}\left(\beta_{1}\right)$ is in the center of $\mathrm{H}^{*}(P, A)$. Hence

$$
\chi^{2 m_{1}+2 m_{2}} \mathrm{H}^{*}(P, M) \subseteq \beta_{1}\left(\chi^{2 m_{2}} \mathrm{H}^{*}(P, M)\right) \subseteq\left(\beta_{1} \beta_{2}\right) \mathrm{H}^{*}(P, M) .
$$

Continuing in this way, we see

$$
\chi^{2 m_{1}+\cdots+2 m_{r}} \mathrm{H}^{*}(P, M) \subseteq \beta_{1} \ldots \beta_{r} \mathrm{H}^{*}(P, M)=0 .
$$

That completes the inductive step, and the proof of Lemma 5.1 .

\section{ACKNOWLEDGMENT}

The author thanks D. J. Benson for some clarifying conversations.

\section{REFERENCES}

[1] J. L. Alperin and M. Broué, Local methods in block theory, Ann. Math. 110 (1979), 143-157. MR0541333 (80f:20010)

[2] J. L. Alperin and L. Evens, Representations, resolutions, and Quillen's dimension theorem, J. Pure Appl. Algebra 22 (1981), 1-9. MR0621284 (82j:20020)

[3] J. L. Alperin and L. Evens, Varieties and elementary abelian groups, J. Pure Appl. Algebra 26 (1982), no. 3, 221-227. MR0678520 (84a:20057)

[4] M. F. Atiyah and I. G. MacDonald, Introduction to Commutative Algebra, Addison-Wesley, 1969. MR0242802 (39:4129)

[5] G. S. Avrunin and L. L. Scott, Quillen stratification for modules, Invent. Math. 66 (1982), 277-286. MR0656624 (83h:20048)

[6] D. J. Benson, Representations and Cohomology I: Basic representation theory of finite groups and associative algebras, Cambridge Studies in Advanced Mathematics 30, Cambridge University Press, 1991. MR 1110581 (92m:20005)

[7] D. J. Benson, Representations and Cohomology II: Cohomology of groups and modules, Cambridge Studies in Advanced Mathematics 31, Cambridge University Press, 1991. MR.1156302 (93g:20099)

[8] H. Cartan and S. Eilenberg, Homological Algebra, Princeton University Press, 1956. MR0077480 (17:1040e)

[9] C. W. Curtis and I. Reiner, Methods of Representation Theory with Applications to Finite Groups and Orders, Volume II, Wiley, 1987. MR0892316 (88f:20002)

[10] K. Erdmann and N. Snashall, On Hochschild cohomology of preprojective algebras, I, II, J. Algebra 205 (1998), 391-412, 413-434. MR 1632808 (99e:16013)

[11] L. Evens, Cohomology of Groups, Oxford University Press, 1991. MR.1144017 (93i:20059)

[12] M. Gerstenhaber, The cohomology structure of an associative ring, Ann. of Math. 78 (1963), 267-288. MR0161898 (28:5102)

[13] E. L. Green, N. Snashall and Ø. Solberg, The Hochschild cohomology ring of a selfinjective algebra of finite representation type, Proc. Amer. Math. Soc. 131 (2003), no. 11, 3387-3393. MR.1990627 (2004c:16013)

[14] M. Linckelmann, Transfer in Hochschild cohomology of blocks of finite groups, Algebras Representation Theory 2 (1999), 107-135. MR1702272 (2000h:20024)

[15] M. Linckelmann, Varieties in block theory, J. Algebra 215 (1999), 460-480. MR.1686201 (2000c:20022)

[16] M. Linckelmann, Quillen stratification for block varieties, J. Pure Appl. Algebra 172 (2002), 257-270. MR.1906878 (2003g:20015)

[17] J. Pakianathan and S. Witherspoon, Hochschild cohomology and Linckelmann cohomology for blocks of finite groups, J. Pure Appl. Algebra 178 (2003), 87-100. MR1947968(2004d:20010) 
[18] D. Quillen, The spectrum of an equivariant cohomology ring I, II, Ann. Math. 94 (1971), 549-572, 573-602. MR0298694 (45:7743)

[19] D. Quillen and B. Venkov, Cohomology of finite groups and elementary abelian subgroups, Topology 11 (1972), 317-318. MR0294506 (45:3576)

[20] J.-P. Serre, Sur la dimension cohomologique des groupes profinis, Topology 3 (1965), 413-420. MR0180619 (31:4853)

[21] S. F. Siegel, Varieties for Hochschild cohomology of group algebras and blocks, unpublished notes, 1997.

[22] S. F. Siegel and S. J. Witherspoon, The Hochschild cohomology ring of a group algebra, Proc. London Math. Soc. 79 (1999), 131-157. MR1687539 (2000b:16016)

[23] S. F. Siegel and S. J. Witherspoon, The Hochschild cohomology ring of a cyclic block, Proc. Amer. Math. Soc. 128 (2000), 1263-1268. MR1691003 (2000j:16016)

[24] N. Snashall and Ø. Solberg, Support varieties and Hochschild cohomology rings, Proc. London Math. Soc. 88 (2004), no. 3, 705-732. MR2044054 (2005a:16014)

[25] A. Suslin, The detection theorem for finite group schemes, to appear in J. Pure Appl. Algebra.

[26] J. Thévenaz, Some remarks on G-functors and the Brauer morphism, J. Reine Angew. Math. 384 (1988), 24-56. MR0929977 (89b:20035)

Department of Mathematics, University of Rochester, Rochester, New York 14627

E-mail address: jonpak@math.rochester.edu

Department of Mathematics, Texas A\&M University, College Station, Texas 77843

E-mail address: sjw@math.tamu.edu

Department of Computer Science, University of Massachusetts, Amherst, MassaCHUSETTS 01003-9264

E-mail address: siegel@cs.umass.edu 